\title{
Modelos do gelo e transições de fase
}

\author{
Ice Models and phase transitions
}

\author{
Everton Artuso*10 \\ ${ }^{1}$ Universidade Federal da Fronteira Sul, Campus Realeza, PR, Brasil.
}

Recebido em 12 de novembro de 2020. Revisado em 03 de fevereiro de 2021. Aceito em 12 de fevereiro de 2021.

Nesse trabalho consideramos modelos estatísticos unidimensionais, em particular os modelos do gelo de seis e oito vértices, cujas funções de partição podem ser escritas em termos de uma matriz de transferência, e que exibem o fenômeno de transição de fases.

Palavras-chave: Modelos do gelo, autovalor maximal, transição de fase.

In this work we consider one-dimensional statistical models, in particular the six and eight vertex ice models, whose partition functions can be written in terms of a transfer matrix, and which exhibit the transition phenomenon of phases.

Keywords: Ice models, maximal eigenvalue, phase transition.

\section{Introdução}

Experimentalmente, a baixas temperaturas, um sistema ferromagnético pode se manter magnetizado indefinidamente - tratando-se de uma magnetização espontânea a campo magnético nulo, uma vez que havendo campo magnético externo aplicado, a transição de fase ferromagnética-paramagnética em questão deixa de existir. Contudo, se a temperatura for gradualmente aumentada, observa-se que existe um valor crítico a partir do qual a magnetização se anula. Tal comportamento indica a presença de transição de fase nesses sistemas. Em tal valor crítico a susceptibilidade magnética, que é uma derivada da energia livre, é infinita. Intuitivamente, em baixas temperaturas há, de certa forma, uma cooperação entre os spins de sítios vizinhos, devido ao acoplamento entre eles, o que resulta em um alinhamento preferencial para o momento de dipolo. Há assim uma fase ordenada para o sistema em temperaturas baixas. Já em temperaturas altas, a agitação térmica consegue destruir o comportamento ordenado do sistema. Os momentos de dipolo assumem direções aleatórias, sendo que, na média, não há uma magnetização resultante. Esta mudança de comportamento caracteriza uma transição de fase.

Transições de fase ocorrem quando há perda de regularidade de uma energia livre com relação a temperatura no limite termodinâmico. Do ponto de vista experimental, é possível distinguir entre dois tipos de transição de fase: as transições de primeira ordem, nas quais ocorre a coexistência de fases, como por exemplo um sólido de alta densidade e um fluido de baixa densidade; e as transições contínuas (de segunda ordem) nas quais

\footnotetext{
* Endereço de correspondência: everton.artuso@uffs.edu.br
}

flutuações e correlações crescem de tal ponto que seja macroscopicamente observável [1].

De uma perspectiva termodinâmica, a compreensão de transições de primeira ordem se dá associando a cada fase uma energia livre. A fase escolhida pelo sistema, dados certos parâmetros externos, é aquela com a menor energia livre de modo que uma transição de fase ocorre quando as energias livres de duas (ou mais) fases são iguais. Mudanças repentinas em quantidades macroscopicamente mensuráveis que ocorrem em transições de primeira ordem são descritas matematicamente como descontinuidades na primeira derivada da energia livre. Já as descontinuidades em derivadas de ordem superior estão relacionadas a transições de fase contínuas (de ordem superior) 1 .

A energia livre é dada pelo logaritmo da função de partição, uma quantidade que normaliza a distribuição de probabilidade de estado estacionário de configurações microscópicas, no limite termodinâmico. De modo a reproduzir corretamente as singularidades na energia livre em transições de primeira ordem, Lee e Yang, em [2] e [3, introduziram uma descrição de transições de fase relativas a zeros da função de partição quando generalizada para o plano complexo de uma quantidade termodinâmica intensiva, ou seja, cuja magnitude é independente do tamanho do sistema. Tais singularidades na energia livre ocorrem somente no limite termodinâmico. A função pressão, abordada no capítulo 2, está conectada, como conceito termodinâmico, a função de partição - conceito da mecânica estatística - e, por consequência, a energia livre no limite termodinâmico.

Um dos modelos que tradicionalmente introduz o método da matriz de transferência é o conhecido modelo de Ising, que em uma dimensão não apresenta transição de fase, mas em maiores dimensões tais transições podem 
ocorrer 4, 5]. Em particular, a técnica da matriz de transferência foi utilizada por Onsager em [6] na solução original do modelo de Ising em duas dimensões.

Os modelos do gelo, originalmente propostos com vistas a explicar a entropia residual do gelo em [7] e a transição de fases no cristal ferroelétrico $\mathrm{KH}_{2} \mathrm{PO}_{4}$ em [8, por sua vez, apresentam transição de fase unidimensional. O modelo de seis vértices foi formulado e resolvido na rede quadrada por E. Lieb na década de 60. Logo depois, Baxter, em 9, resolveu o modelo de oito vértices na rede quadrada. Essas soluções, que dependem da formulação do problema em termos de uma matriz de transferência, estão bem descritas no artigo de revisão [10]. Tais modelos foram revisitados, também via matriz de transferência, em 11 . A técnica da matriz de transferência será utilizada a fim de mostrar que a transição de fase está associada à degenerescência do seu autovalor máximo.

Atualmente, modelos similares aos modelos do gelo (ou oriundos deles) ainda são estudados. Conforme o artigo de revisão 12, podemos citar os modelos do gelo artificiais e os modelos do tipo 'spin ice' magnético. Tal artigo aborda a conexão entre a regra do gelo e a frustração geométrica (a qual se refere à incapacidade de um sistema de satisfazer interações concorrentes na presença de restrições espaciais), além de apresentar a origem de tais técnicas na matéria condensada 'soft' e exemplificar sua atuação possibilitando a criação de novas e exóticas fases da matéria, potencialmente direcionando-as para aplicações tecnológicas relacionadas a memória e dispositivos lógicos que se baseiam no movimento de defeitos topológicos

Uma curiosidade matemática interessante é que, conforme [10], a solução do modelo do gelo também dá o número de formas de colorir todas as faces de um reticulado quadrado com três cores de modo que não haja duas faces adjacentes com a mesma cor.

Inicialmente apresentamos os modelos de Ising, logo enunciamos o Teorema de Perron-Frobenius e um Teorema auxiliar e depois os aplicamos, juntamente com o formalismo da matriz de transferência, aos modelo do gelo de seis e oito vértices.

\section{Modelo de Ising}

Consideremos uma amostra de algum material cujos átomos estão arranjados em uma estrutura regular cristalina. Suponhamos que cada um desses átomos carrega um momento magnético (algo como um ímã ligado a cada átomo) chamado de spin. Assumamos que cada spin tem a tendência de se alinhar com seus vizinhos e que, inicialmente, esteja orientados aleatoriamente, como na Figura 1. Nessa seção, nos baseamos em [4].

Se o material é exposto a um campo magnético externo apontando numa direção específica, então um tipo de ordem aparece: os spins tendem a se alinhar com o campo, e assim apontando na mesma direção. Se diminuímos devagar a intensidade do campo externo até zero, dois casos podem ocorrer.

$\mathrm{Na}$ Figura 2, a ordem global é progressivamente perdida conforme o campo decresce, chegando a zero, quando os spins tornam-se desordenados novamente, como no seu estado inicial. Tal comportamento é chamado paramagnético.

Esse fenômeno pode ser medido quantitativamente ao se introduzir a magnetização, que é a média dos spins, projetada na direção do campo magnético. Para o paramagneto, conforme o campo decresce de um valor positivo para zero (mantendo a direção fixada), ou similarmente, se ele cresce de um valor negativo para zero, a magnetização tende a zero, como na Figura 3

Ainda outro cenário é possível: como o campo externo decresce, a ordem global decresce, mas a interação local entre os spins é forte o suficiente para manter o material em estado de magnetização global mesmo depois que o campo externo tenha alcançado zero. Tal comportamento é chamado ferromagnetismo, representado na Figura 4

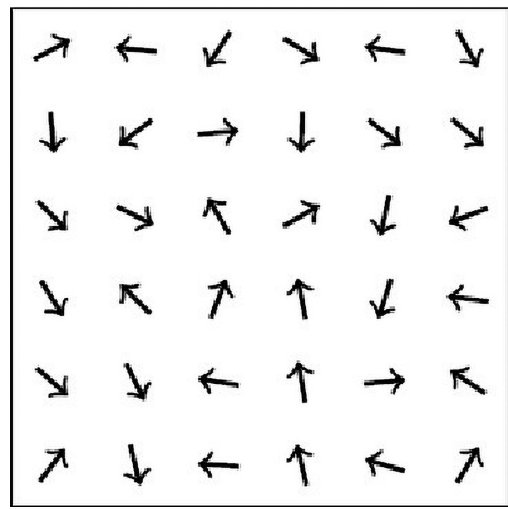

Figura 1: 4] Estado inicial.

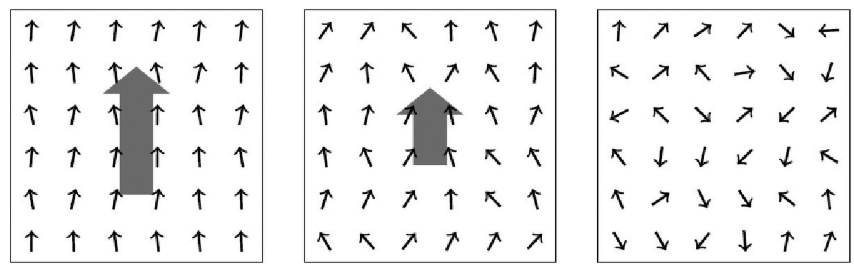

Figura 2: 4] Campo magnético decrescente.

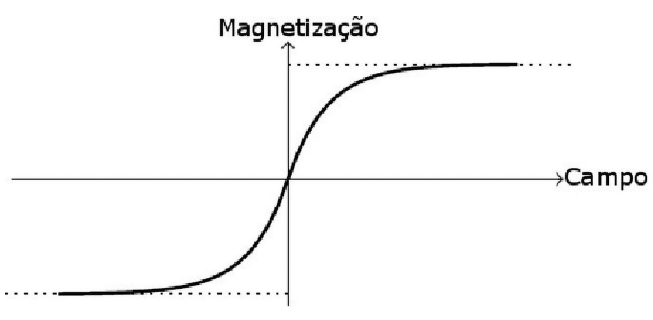

Figura 3: [4]. 


\begin{tabular}{|c|c|c|c|c|c|c|c|c|c|c|}
\hline & $\uparrow$ & $\uparrow \uparrow$ & T & $\uparrow$ & & $\uparrow$ & $\uparrow$ & & 7 & \\
\hline$\uparrow$ & $\uparrow$ & $\uparrow \uparrow$ & 1 & $\uparrow$ & $\uparrow$ & $\uparrow$ & $\uparrow$ & & $t$ & 7 \\
\hline$\uparrow$ & $\uparrow$ & & & $\uparrow$ & $\uparrow$ & $\uparrow$ & & & & 7 \\
\hline$\uparrow$ & $\uparrow$ & $\uparrow \uparrow$ & 1 & $\uparrow$ & $\uparrow$ & $\uparrow$ & & & & $\uparrow$ \\
\hline$\uparrow$ & $\uparrow$ & $\uparrow \uparrow$ & & $\uparrow$ & $\uparrow$ & $\uparrow$ & † & $\uparrow$ & & $\uparrow$ \\
\hline$\uparrow$ & $\uparrow$ & $\uparrow \uparrow$ & & $\uparrow \uparrow$ & $\uparrow$ & $\uparrow$ & $\uparrow$ & $\uparrow$ & $\uparrow$ & $\uparrow$ \\
\hline
\end{tabular}

Figura 4: 4] Ferromagnetismo.

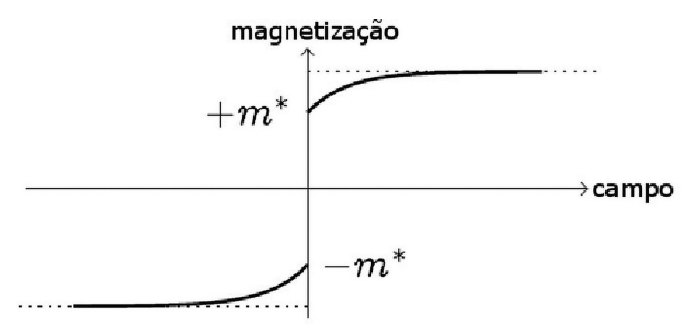

Figura 5: 4] Magnetização espontânea.

Um ferromagneto exibe, portanto, magnetização espontânea, que é uma ordem global produzida mesmo com a ausência de um campo magnético externo. O valor da magnetização espontânea, $\pm m^{*}$, depende da forma em que o campo externo se aproxima de zero (de $>0$ ou $<0$ ), representado na Figura 5 .

Usando o processo descrito, pode-se, a princípio, preparar um material ferromagnético com magnetização espontânea em uma direção arbitrária aplicando o campo magnético nessa direção, e fazê-lo decrescer lentamente a zero. Pode-se observar ainda que, na Figura 5 , quando o campo vai para zero, a magnetização tem uma descontinuidade e salta de um valor estritamente positivo para uma valor estritamente negativo, o que representa uma transição de fase de primeira ordem.

O modelo de Ising é um modelo simples cujo objetivo é reproduzir as propriedades descritas acima. A principal simplificação é assumir que os spins são restritos a uma direção particular, apontando para cima ou para baixo. Apesar de ser um modelo simples, é útil na descrição de outros sistemas (tais como um gás) que, com as devidas simplificações, pode ser mapeado pelo modelo de Ising. Para mais detalhes, veja 4].

A energia de uma configuração $\omega \in \Omega_{V}:=\{-1,1\}^{V}$, onde -1 e 1 são os spins e $V$ é um conjunto finito (para mais detalhes veja o Apêndice A no material suplementar), é obtida através da soma das interações sobre todos os pares, e pela adição da interação de cada spin com o campo magnético $h$

$$
H:=-\beta \sum_{i \sim j} \varsigma_{i} \varsigma_{j}-h \sum_{i} \varsigma_{i},
$$

onde $\varsigma_{i}: \Omega_{V} \rightarrow\{-1,1\}$ definida por $\varsigma_{i}(\omega):=\omega_{i}$ é uma variável aleatória e dá o valor do spin no vértice $i$. A função $H$ é também conhecida como o Hamiltoniano. De acordo com a Mecânica Estatística, a probabilidade

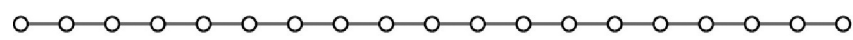

Figura 6: [4] Caixa unidimensional.

de observação do sistema na configuração $\omega$ é dada por

$$
\mu(\omega):=\frac{1}{Z} \exp (-H(\omega))
$$

onde $e^{-H(\omega)}$ é conhecido como peso de Boltzmann. A constante de normalização

$$
Z:=\sum_{\omega \in \Omega_{V}} \exp (-H(\omega))
$$

desempenha um importante papel na teoria, e é conhecida como função de partição.

Dois pontos $i, j \in \mathbb{Z}$ são vizinhos próximos se $|j-i|=1$, que denotamos por $i \sim j$. Denotamos por $V_{N}$ uma caixa de tamanho linear $N$ unidimensional,

$$
V_{N}:=\{x \in \mathbb{Z}: 0 \leq x<N\},
$$

representada na Figura 6. Para mais detalhes, veja [4].

O objetivo é estudar o modelo de Ising em uma caixa grande $V_{N}$. Assim, iremos considerar o modelo através de uma sequência de caixas crescentes $V_{1}, V_{2}, \ldots, V_{N}, \ldots$, e descrever o seu comportamento no limite termodinâmico, isto é, quando $N \rightarrow \infty$. A energia livre é dada pelo limite (quando este existir)

$$
\psi(\beta, h):=\lim _{N \rightarrow \infty} \frac{1}{\left|V_{N}\right|} \log Z_{V_{N}, \beta, h}
$$

Uma transição de fase pode ser interpretada como uma singularidade da energia livre em alguma das suas variáveis. Em geral, o comportamento assintótico (quando $N \rightarrow \infty$ ) do sistema descrito pela distribuição de Gibbs em uma caixa suficientemente grande pode ser relacionado com as propriedades analíticas da energia livre em $h$. Assumindo que o limite e as derivadas podem ser intercambiados (o que acontece sob certas circunstâncias),

$$
\frac{\partial \psi_{\beta}(h)}{\partial h}=m_{\beta}(h)
$$

e portanto, a magnetização média está relacionada com a derivada da energia livre.

A energia livre $\psi_{\beta}(h)$ do Modelo de Ising unidimensional é analítica em $h$ em todas as temperaturas, conforme demonstrado no Apêndice A do Material Suplementar.

Seja $T_{N}$ o grafo obtido por ligar $N-1$ com $0 \mathrm{em}$ $V_{N}=\{0,1, \ldots, N-1\}$ como um toro (Figura 7). Formalmente, $T_{N}$ é obtido de $V_{N}$ adicionando uma aresta entre $N-1$ e 0 . Ao transformarmos $V_{n}$ em $T_{n}$, estamos caracterizando as condições de contorno periódicas.

A vantagem de se trabalhar com $T_{N}$ ao invés de $V_{N}$ é que $Z_{T_{N}, \beta, h}$ pode ser escrito como o traço de uma 


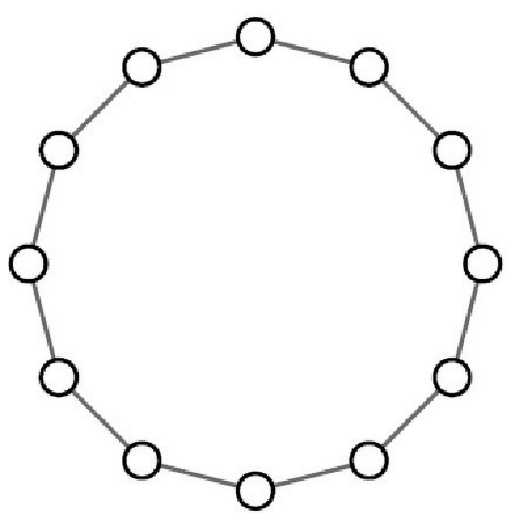

Figura 7: 4] Envolvendo $V_{N}$ em um toro $T_{N}$

matriz $2 \times 2$. De fato, assumindo a condição de contorno periódica $\omega_{N}=\omega_{0}$, temos

$$
Z_{T_{N}, \beta, h}=\sum_{\substack{\omega_{j}= \pm 1 \\ j \in\{0, \ldots, N-1\}}} \prod_{i=0}^{N-1} A_{\omega_{i}, \omega_{i+1}},
$$

onde os números $A_{+,+}=e^{\beta+h}, A_{+,-}=e^{-\beta+h}, A_{-,+}=$ $e^{-\beta-h}$ e $A_{-,-}=e^{\beta-h}$ podem ser colocados na forma de uma matriz, chamada matriz de transferência:

$$
A=\left(\begin{array}{cc}
e^{\beta+h} & e^{-\beta+h} \\
e^{-\beta-h} & e^{\beta-h}
\end{array}\right) .
$$

Uma observação útil é que $Z_{T_{N}, \beta, h}$ pode ser interpretado com o traço da $N$-ésima potência de $A$ :

$$
Z_{T_{N}, \beta, h}=\sum_{\omega_{0}= \pm 1}\left(A^{N}\right)_{\omega_{0}, \omega_{0}}:=\operatorname{tr}\left(A^{N}\right) .
$$

$A$ tem dois dois autovalores $\lambda_{+}>\lambda_{-}$dados por

$$
\lambda_{ \pm}=e^{\beta} \cosh (h) \pm \sqrt{e^{2 \beta} \cosh ^{2}(h)-2 \operatorname{senh}(2 \beta)},
$$

e como $\lambda_{+}>\lambda_{-}$, tomando o limite termodinâmico, temos $\psi_{\beta}(h)=\log \lambda_{+}$, e para todo $\beta \geq 0$, e todo $h \in \mathbb{R}$, a energia livre $\psi_{\beta}(h)$ existe e é igual a

$\psi_{\beta}(h)=\log \left\{e^{\beta} \cosh (h)+\sqrt{e^{2 \beta} \cosh ^{2}(h)-2 \operatorname{senh}(2 \beta)}\right\}$,

que é uma função analítica. Em outros termos, o fato da energia livre não apresentar nenhuma singularidade no limite termodinâmico (o que é assegurado pelos Teoremas 3.1 e 3.2 e a estabilidade de um único autovalor simples e maximal) garante que o modelo de Ising unidimensional não apresenta coexistência de fases, não havendo, portanto, nenhuma transição. Para mais detalhes, consulte [4] e [5].

\section{Matriz de Perron-Frobenius}

Muitas vezes, transições de fase podem ser detectadas por meio de singularidades na energia livre no limite termodinâmico. A energia livre, por sua vez, coincide com o maior autovalor (também dependente da temperatura e do campo magnético) da matriz de transferência. As ferramentas essenciais para a execução dessa análise são o Teorema de Perron-Frobenius e outro teorema que garante a analiticidade dos autovalores da matriz de transferência.

Uma matriz $M=\left(m_{i, j}\right)_{0 \leq i, j \leq s-1}$ é dita não negativa se cada uma das suas entradas for não negativa. Em outros termos, para todo $i, j \in\{0, \ldots, s-1\}, m_{i j} \geq 0$. Uma matriz não negativa $M$ é dita irredutível se, para todo $i, j$ existe algum $k \geq 1$ tal que $m_{i j}^{(k)}>0$, denotando por $\left(m_{i j}^{(k)}\right)$ as entradas da $k$-ésima potência de $M$, a saber, $M^{k}$. Ela é dita primitiva se $M^{k}$ é positiva para algum $k \geq 1$, isto é, todos os $m_{i j}^{(k)}>0$ para o mesmo $k$, e o menor $k$ tal que isso aconteça é chamado de índice de primitividade de $M$.

Teorema 3.1 (Perron-Frobenius). [13] Seja $M$ uma matriz primitiva. Então

(i) $M$ admite um autovalor $\theta$ tal que $|\lambda|<\theta$ para quaisquer outros autovalores $\lambda$ de $M$.

(ii) Existe um autovetor com todas as entradas positivas associado ao autovalor $\theta$.

(iii) $\theta$ é um autovalor simples, ou seja, tem multiplicidade algébrica um.

Demonstração: Veja Teorema 5.4 de [13.

O Teorema de Perron-Frobenius apresenta várias aplicações, muitas das quais apresentadas em [14, onde também encontramos diversas demonstrações do referido Teorema.

\subsection{Transição de Fase}

O Teorema de Perron-Frobenius garante que o autovalor maximal de matrizes primitivas é simples e estritamente positivo. Tal fato gera consequências muito importantes na teoria da termodinâmica de sistemas físicos, de modo que em muitos sistemas de spins unidimensionais, a pressão - função analítica que depende da temperatura - coincide com o logaritmo natural de tal autovalor. O fenômeno da transição de fase se dá, matematicamente, através da perda de regularidade da função pressão, ou seja, quando alguma de suas infinitas derivadas se torna descontínua.

Embora fundamental, o Teorema de Perron-Frobenius não é suficiente para mostrar se há ou não transição de fase quando as entradas da matriz de transferência dependem do parâmetro da temperatura $\beta$. Nesse caso temos uma família de matrizes dependendo da temperatura, $M(\beta)$, e precisamos de outro resultado válido para matrizes analíticas em $\beta$ (ou seja, todos os seus elementos são funções analíticas de $\beta$ ).

Teorema 3.2. [15] Para todo $\beta$ em um conjunto simplesmente conexo $D \subset \mathbb{C}$, seja $M(\beta)$ um operador linear em um espaço vetorial $X$ n-dimensional $(M(\beta)$ 
é uma matriz complexa $n \times n)$. Se $M(\beta)$ for analítica em $D$ então cada um dos s autovalores (aqueles que não aumentam ou diminuem em número) de $M(\beta)$ tem multiplicidade constante e pode ser expresso como uma função analítica em $D, \lambda_{j}(\beta), j \in\{1, \ldots, s\}$.

Demonstração: Veja Teorema 1.8 no Capítulo II de [16].

Assim, para uma matriz de transferência não negativa irredutível $M(\beta)$ cujos elementos são funções analíticas em uma vizinhança do eixo real positivo, $\beta>0$, os Teoremas 3.1 e 3.2 garantem que o autovalor maximal (portanto a energia livre) é uma função analítica de $\beta$, para todo $\beta>0$.

\section{Modelos do Gelo}

Os modelos do gelo são ferramentas muito importantes na introdução dos modelos ferroelétricos. Algumas substâncias, tais como o gelo e $C O$, aparentemente tem uma entropia residual mesmo em temperaturas muito baixas. No caso do gelo a entropia a $10^{\circ} \mathrm{K}$ é $0,82 \pm$ $0,05 \mathrm{cal} /{ }^{\circ} \mathrm{K}-$ mole.

Conforme a simplificação de Slater em 8 com relação a distribuição dos hidrogênios, existe sempre um, e somente um, átomo de hidrogênio em cada ligação (a ponte funciona como um duplo poço de potencial, com dois mínimos próximos dos vértices nas suas extremidades); e existe precisamente dois átomos de hidrogênio próximos (e dois átomos de hidrogênio distantes) de um dado vértice. Tais restrições são plausíveis física e quimicamente e garantem a neutralidade elétrica local, sendo conhecidas como regras do gelo.

No cristal do gelo cada molécula de água forma quatro ligações de hidrogênio. Se a distribuição de íons de hidrogênio obedece as regras do gelo, podemos escrever a entropia residual, $S$, como

$$
S=k \log Z_{0}
$$

onde $k$ é a constante de Boltzmann, o logaritmo tem base exponencial e $Z_{0}$ é a função de partição

$$
Z=\sum_{\text {estados }} e^{-\beta E}
$$

com todas as energias nulas. Além disso, $Z_{0}$ representa o número de configurações de hidrogênios do cristal consistentes com a regra do gelo. A função de partição apresentada na equação (13) consiste na soma sobre todos os estados de distribuições de hidrogênio, $\beta=\frac{1}{k T}$ e $E$ é a energia do cristal para um dado estado.

Conforme [10, o qual revisa resultados de, entre outros, 17 20, em um cristal de gelo os oxigênios estão situados nos vértices do reticulado, enquanto os hidrogênios são posicionados nas arestas do reticulado

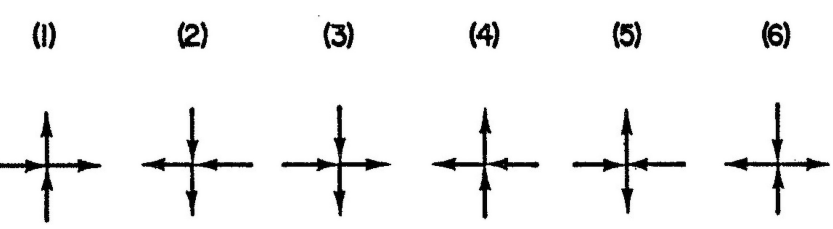

Figura 8: [10] As seis configurações permitidas pelas regras de gelo.
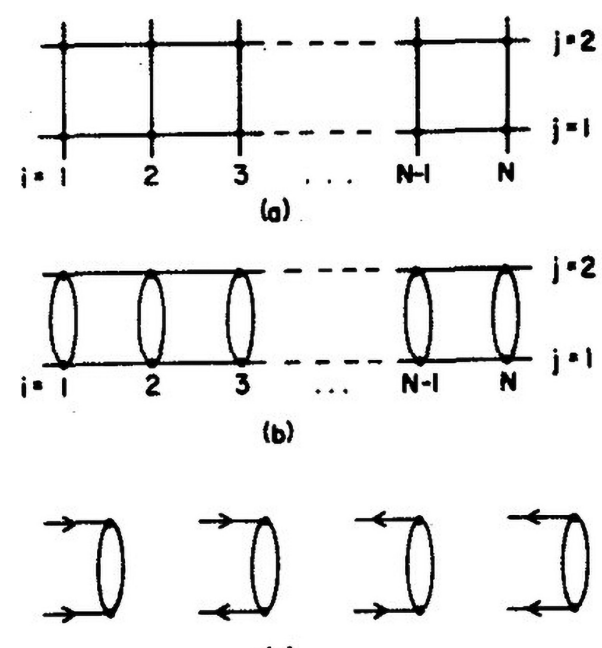

(c)

Figura 9: 11] a) Geometria dos análogos unidimensionais de [11] b) as condições periódicas de contorno na direção vertical evidenciam que cada vértice está ligado aos seus vizinhos através de quatro pontes de hidrogênio; c) os quatro valores possíveis de um estado $\phi_{i}$.

podendo ser especificados por setas sobre essas arestas. Então $Z_{0}$ é o número de formas de direcionar os setas do reticulado de modo que existam sempre duas setas apontando para fora e duas setas apontando para dentro em cada sítio do reticulado. Os seis possíveis estados de cada vértice são ilustrados na Figura 8

Os modelos construídos em 11] são dados por uma cadeia dupla de $N$ vértices na direção horizontal e $M=2$ vértices na direção vertical, conforme Figura 9 a, adotando-se condições periódicas de contorno em ambas as direções vertical e horizontal. Cada vértice está ligado a quatro outros através de pontes de hidrogênio e na Figura 9b estão representadas as condições de contorno vertical. As ligações de hidrogênio horizontais são as únicas a serem representadas e seja $\phi_{i}$ o estado do par de ligações horizontais entre os vértices $i$ e $i+1$. Para qualquer $i$, o estado $\phi_{i}$ é totalmente especificado e, conforme a Figura 9c de [1], há apenas quatro valores distintos de $\phi_{i}$.

\subsection{Modelo de seis vértices}

Vamos considerar um modelo de seis vértices generalizado, conforme [11, com as energias $\varepsilon_{1} \geq 0$ para as 

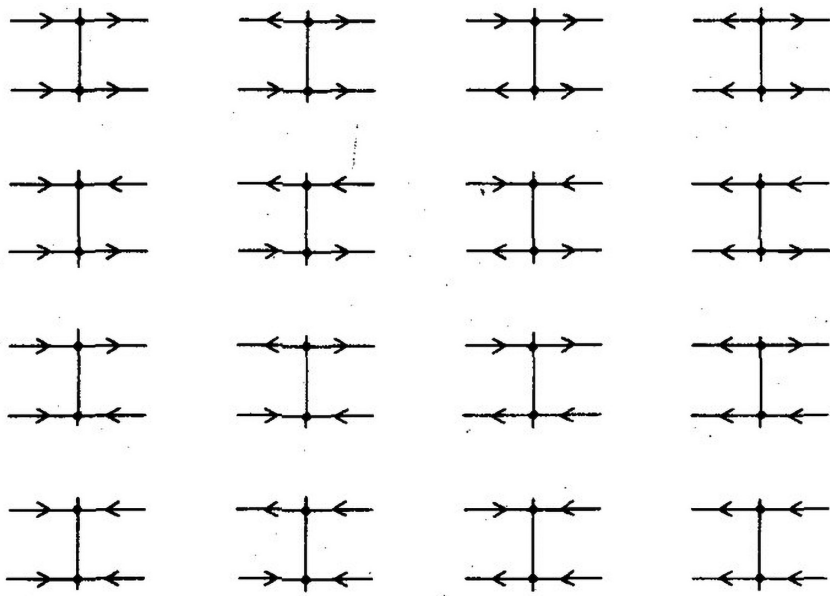

Figura 10: 11 Representação esquemática da matriz de transferência $(4 \times 4)$ para os quatro valores possíveis de um estado $\phi_{i}$. Na primeira linha temos $1 \rightarrow 1,2 \rightarrow 1,3 \rightarrow 1 \mathrm{e}$ $4 \rightarrow 1$ ( 2 e 3 trocam de lugar na Figura 9 f).

configurações (1) e (2), $\varepsilon_{2} \geq 0$ para as configurações (3) e (4) e $\varepsilon_{3} \geq 0$ para as configurações (5) e (6). A construção das entradas da matriz de transferência se dará conforme as configurações aceitáveis em cada um dos valores possíveis de um estado $\phi_{i}$ da Figura 9r, os quais são representados esquematicamente na Figura 10.

O cálculo de cada entrada da matriz de transferência é realizado no Apêndice B do Material Suplementar, e a matriz de transferência $\mathbb{T}$ é dada por

$$
\mathbb{T} \equiv\left(\begin{array}{cc}
e^{-2 \beta \varepsilon_{1}}+e^{-2 \beta \varepsilon_{2}} & 0 \\
0 & 2 e^{-\beta\left(\varepsilon_{1}+\varepsilon_{2}\right)} \\
0 & e^{-2 \beta \varepsilon_{3}} \\
0 & 0 \\
0 & 0 \\
e^{-2 \beta \varepsilon_{3}} & 0 \\
2 e^{-\beta\left(\varepsilon_{1}+\varepsilon_{2}\right)} & 0 \\
0 & e^{-2 \beta \varepsilon_{1}}+e^{-2 \beta \varepsilon_{2}}
\end{array}\right) .
$$

\subsubsection{Autovalores}

Os autovalores de $\mathbb{T}$ são os números $\lambda$ (reais nesse caso, uma vez que a matriz é simétrica) tais que $\mathbb{T} v=\lambda v$ para algum vetor $v \in \mathbb{R}^{4}, \operatorname{logo}\left(\mathbb{T}-\lambda \mathbb{I}_{4}\right) v=0$ aceita soluções não triviais apenas se a matriz $\mathbb{T}-\lambda \mathbb{I}_{4}$ for não invertível, ou seja, quando $\operatorname{det}\left(\mathbb{T}-\lambda \mathbb{I}_{4}\right)=0$. A análise dos autovalores também é realizada no Apêndice B do Material Suplementar. Em resumo, temos

$$
\begin{aligned}
& \lambda_{1}=\lambda_{2}=e^{-2 \beta \varepsilon_{1}}+e^{-2 \beta \varepsilon_{2}}, \\
& \lambda_{3}=2 e^{-\beta\left(\varepsilon_{1}+\varepsilon_{2}\right)}-e^{-2 \beta \varepsilon_{3}}
\end{aligned}
$$

e

$$
\lambda_{4}=2 e^{-\beta\left(\varepsilon_{1}+\varepsilon_{2}\right)}+e^{-2 \beta \varepsilon_{3}} .
$$

Para compararmos $\lambda_{1}$ com $\lambda_{4}$, os dois maiores autovalores, temos dois casos. (i) $\lambda_{1} \geq \lambda_{4}$ :

Se $\lambda_{1} \geq \lambda_{4}$ então temos $-\beta \varepsilon_{1} \geq \log (2)-\beta \varepsilon_{j}$, onde o logaritmo tem base natural, $\operatorname{logo} \beta\left(\varepsilon_{j}-\varepsilon_{1}\right) \geq$ $\log (2)$ e assim

$$
\beta \geq \frac{\log (2)}{\varepsilon_{j}-\varepsilon_{1}}
$$

(ii) $\lambda_{1} \leq \lambda_{4}$ :

Se $\lambda_{1} \leq \lambda_{4}$ então temos $-\beta \varepsilon_{1} \leq \log (2)-\beta \varepsilon_{i}$, onde o logaritmo tem base natural, $\log o \beta\left(\varepsilon_{i}-\varepsilon_{1}\right) \leq \log (2)$ e assim

$$
\beta \leq \frac{\log (2)}{\varepsilon_{i}-\varepsilon_{1}}
$$

Para maiores detalhes, veja o Apêndice B do Material Suplementar.

\subsubsection{Transição de fase}

Uma vez que a $n$-ésima potência sua matriz de transferência tem entradas nulas, para qualquer que seja o $n$, não há garantias de um único autovalor maximal por parte do Teorema de Perron-Frobenius. Em vez disso, há sim uma alternância entre os autovalores na posição maximal, o que configura uma quebra de analiticidade da energia livre de Gibbs, ou seja, uma transição de fase. Em outros termos, para termos uma transição de fase, o que significa que a energia livre, dado que os autovalores são positivos e funções analíticas de $\beta$, é não analítica em algum ponto, devemos ter dois autovalores se cruzando em certo $\beta_{c}$.

No limite termodinâmico, resta somente o maior autovalor e, para $N \rightarrow \infty$, a energia livre é dada por

$$
f \equiv \frac{1}{N} F \equiv-\frac{1}{\beta N} \log Z_{N}=-\frac{1}{\beta} \log \max _{i \in\{1,2,3,4\}}\left(\lambda_{i}\right)
$$

onde o logaritmo tem base natural e

$$
Z_{N}=\operatorname{tr}\left(\mathbb{T}^{N}\right)
$$

Como os autovalores $\lambda_{1}$ e $\lambda_{4}$ se cruzam em algum ponto $\beta_{c} \in\left[\min \left(\frac{\log (2)}{\varepsilon_{i}-\varepsilon_{1}}, \frac{\log (2)}{\varepsilon_{j}-\varepsilon_{1}}\right), \max \left(\frac{\log (2)}{\varepsilon_{i}-\varepsilon_{1}}, \frac{\log (2)}{\varepsilon_{j}-\varepsilon_{1}}\right)\right]$,

conforme as Equações (18) e (19), há nesse ponto uma transição de fase. Caso $\varepsilon_{2}=\varepsilon_{3}$, então a transição de fase se dá em

$$
\beta_{c}=\frac{\log (2)}{\varepsilon_{2}-\varepsilon_{1}},
$$

como ilustra a Figura 11 .

\subsection{Modelo de oito vértices}

O modelo de oito vértices é definido pela adição de uma energia $\varepsilon_{4}>0$ associada às configurações de vértices dos tipos (7) e (8) (Figura 12) ao modelo de seis vértices. 


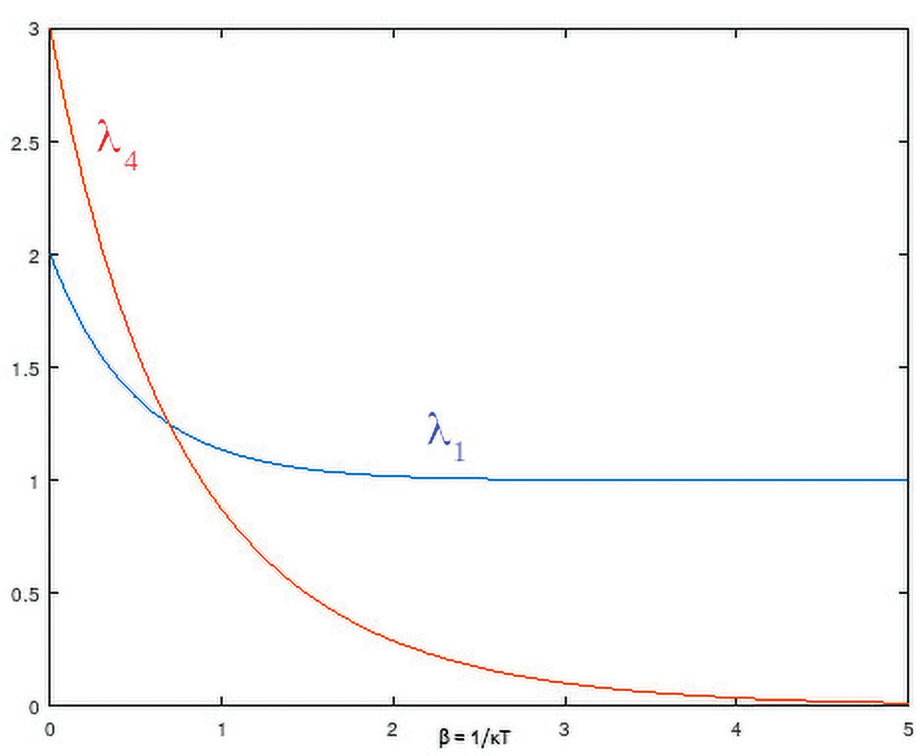

Figura 11: Autovalores $\lambda_{1}$ e $\lambda_{4}$ para $\varepsilon_{1}=0$ e $\varepsilon_{2}=\varepsilon_{3}=1$. A transição de fase ocorre em $\beta=\log (2)$, quando $\lambda_{4}$ deixa de ser e $\lambda_{1}$ se torna o autovalor maximal.

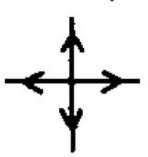

(7)

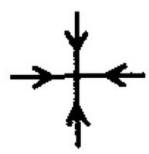

(8)
Figura 12: 11] As configurações (7) e (8), juntamente com as seis configurações permitidas pelas regras de gelo da Figura 8 definem o chamado modelo de oito vértices.

A construção da matriz de transferência para esse caso é muito similar ao caso de seis vértices, e é dada por $\mathbb{T}^{\prime}$ na Equação (24):

$$
\mathbb{T}^{\prime}=\left(\begin{array}{cc}
e^{-2 \beta \varepsilon_{1}}+e^{-2 \beta \varepsilon_{2}} & 0 \\
0 & 2 e^{-\beta\left(\varepsilon_{1}+\varepsilon_{2}\right)} \\
0 & e^{-2 \beta \varepsilon_{3}}+e^{-2 \beta \varepsilon_{4}} \\
2 e^{-\beta\left(\varepsilon_{3}+\varepsilon_{4}\right)} & 0 \\
0 & 2 e^{-\beta\left(\varepsilon_{3}+\varepsilon_{4}\right)} \\
e^{-2 \beta \varepsilon_{3}}+e^{-2 \beta \varepsilon_{4}} & 0 \\
2 e^{-\beta\left(\varepsilon_{1}+\varepsilon_{2}\right)} & 0 \\
0 & e^{-2 \beta \varepsilon_{1}}+e^{-2 \beta \varepsilon_{2}}
\end{array}\right) .
$$

Note que se $\varepsilon_{4} \rightarrow \infty$ então $e^{-\beta \varepsilon_{4}} \rightarrow 0$ e a matriz $\mathbb{T}^{\prime}$ se resume a matriz $\mathbb{T}$ do modelo de seis vértices.

\subsubsection{Autovalores}

Da mesma forma como calculamos os autovalores de $\mathbb{T}$, calculamos os de $\mathbb{T}^{\prime}$ no Apêndice $B$ do Material Suplementar. Tais autovalores são os números $\lambda$ (reais nesse caso, uma vez que a matriz é simétrica) tais que $\mathbb{T}^{\prime} v=\lambda v$ para algum vetor $v \in \mathbb{R}^{4}, \operatorname{logo}\left(\mathbb{T}^{\prime}-\lambda \mathbb{I}_{4}\right) v=0$ aceita soluções não triviais apenas se a matriz $\mathbb{T}^{\prime}-\lambda \mathbb{I}_{4}$ for não invertível, ou seja, quando $\operatorname{det}\left(\mathbb{T}^{\prime}-\lambda \mathbb{I}_{4}\right)=0$. Dessa forma, temos

$$
\begin{aligned}
& \lambda_{1}=e^{-2 \beta \varepsilon_{1}}+e^{-2 \beta \varepsilon_{2}}+2 e^{-\beta\left(\varepsilon_{3}+\varepsilon_{4}\right)}, \\
& \lambda_{2}=e^{-2 \beta \varepsilon_{1}}+e^{-2 \beta \varepsilon_{2}}-2 e^{-\beta\left(\varepsilon_{3}+\varepsilon_{4}\right)}, \\
& \lambda_{3}=2 e^{-\beta\left(\varepsilon_{1}+\varepsilon_{2}\right)}+e^{-2 \beta \varepsilon_{3}}+e^{-2 \beta \varepsilon_{4}} \\
& \lambda_{4}=2 e^{-\beta\left(\varepsilon_{1}+\varepsilon_{2}\right)}-e^{-2 \beta \varepsilon_{3}}-e^{-2 \beta \varepsilon_{4}} .
\end{aligned}
$$

Como $e^{-\beta \varepsilon_{k}}>0$ para todo $\varepsilon_{k} \in \mathbb{R}, k=3$, 4 , temos claramente

$$
\lambda_{1}>\lambda_{2} \text { e } \lambda_{3}>\lambda_{4}
$$

\subsubsection{Transição de fase}

A existência de uma transição de fases dependerá se $\lambda_{1}$ ou $\lambda_{3}$ é o autovalor maximal, para cada $\beta>0$, e se houver tal transição, esta ocorrerá em alguma temperatura crítica tal que $\lambda_{1}=\lambda_{3}$, ou seja,

$$
\left(e^{-\beta \varepsilon_{1}}-e^{-\beta \varepsilon_{2}}\right)^{2}=\left(e^{-\beta \varepsilon_{3}}-e^{-\beta \varepsilon_{4}}\right)^{2} .
$$

Como temos quatro variáveis livres, vamos simplificar a análise fazendo uma escolha particular de energias que, conforme [1], correspondem fisicamente ao modelo de Slater para o $\mathrm{KH}_{2} \mathrm{PO}_{4}$ com a adição das configurações duplamente ionizadas. Assim, sejam $\varepsilon_{1}=0, \varepsilon_{2}=\varepsilon_{3}=$ $\varepsilon>0$ e $\varepsilon_{4}=n \varepsilon$, com $n>0$, e neste caso, a Equação (30) se torna

$$
1-e^{-\beta \varepsilon}= \pm\left(e^{-\beta \varepsilon}-e^{-n \beta \varepsilon}\right)
$$

e os autovalores $\lambda_{1}$ e $\lambda_{3}$ se tornam

$$
\begin{gathered}
\lambda_{1}=1+e^{-2 \beta \varepsilon}+2 e^{-\beta(n+1) \varepsilon}, \\
\lambda_{3}=2 e^{-\beta \varepsilon}+e^{-2 \beta \varepsilon}+e^{-2 n \beta \varepsilon} .
\end{gathered}
$$

Conforme análise realizada no Apêndice B do Material Suplementar, para que haja transição de fase, $n$ deverá ser obrigatoriamente maior do que 2. Em tais circunstâncias, $\lambda_{1}$ é o maior autovalor para temperaturas baixas $\left(\beta>\beta_{c}\right)$ e $\lambda_{3}$ é o maior autovalor para temperaturas altas $\left(\beta<\beta_{c}\right)$, e a transição de fase é de primeira ordem. A Figura 13 apresenta um caso particular.

Por outro lado temos, para $n \leq 2$, um único autovalor maximal, a saber, $\lambda_{1}$, conforme apresentado na Figura 14, o que torna a energia livre do modelo uma função analítica.

No limite termodinâmico, para $N \rightarrow \infty$, a energia livre é dada por

$$
f \equiv \frac{1}{N} F \equiv-\frac{1}{\beta N} \log Z_{N}=-\frac{1}{\beta} \log \max _{i \in\{1,3\}}\left(\lambda_{i}\right)
$$

onde o logaritmo tem base natural e

$$
Z_{N}=\operatorname{tr}\left(\mathbb{T}^{N}\right)
$$

Para $n \leq 2$, a Equação (34) é analítica. Já para $n>2$, não é. 


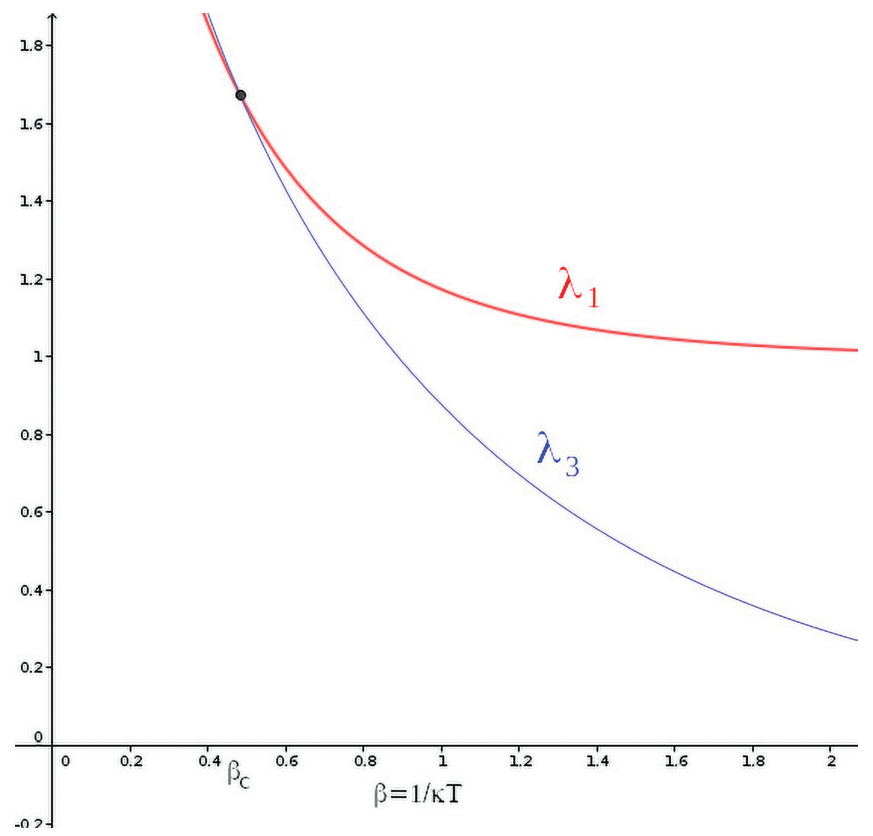

Figura 13: Autovalores $\lambda_{1}$ (Equação (32)) e $\lambda_{3}$ (Equação (33)) para $\varepsilon=1$ e $n=3$. A transição de fase ocorre em $\beta \approx 0,48$, quando $\lambda_{3}$ deixa de ser e $\lambda_{1}$ se torna o autovalor maximal.

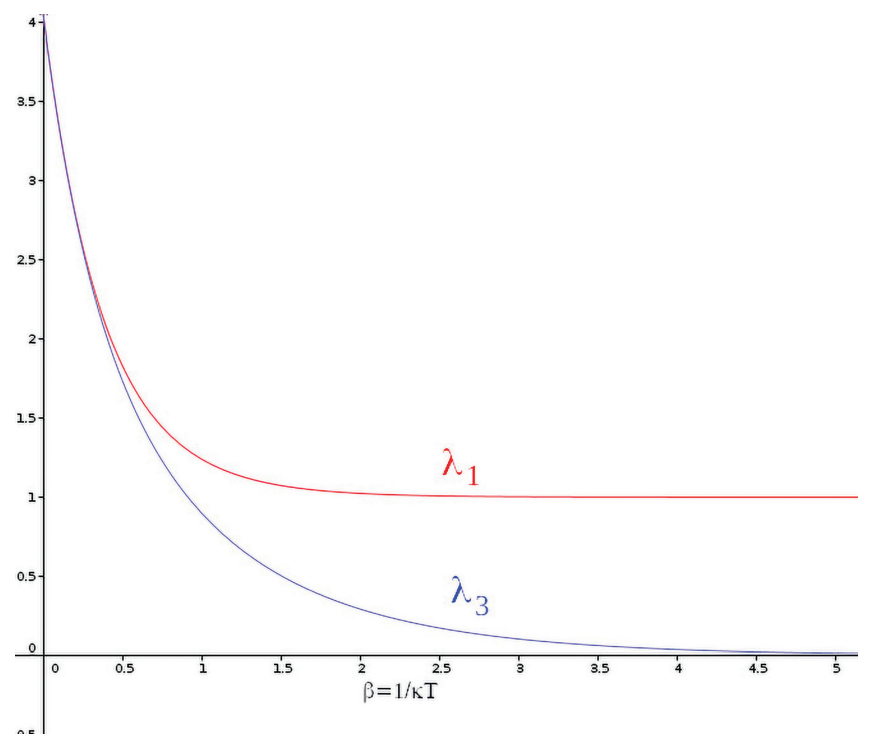

Figura 14: Autovalores $\lambda_{1}$ (Equação (32)) e $\lambda_{3}$ (Equação (33)) para $\varepsilon=1$ e $n=2$. A transição de fase não ocorre e $\lambda_{1}$ é o autovalor maximal.

\section{Discussão e Considerações Finais}

Apresentamos resultados sobre o tratamento de transições de fase via teoria da matriz de transferência, iniciando com o modelo de Ising e mostrando porque não há transição de fase, e apresentando os modelos do gelo de seis vértices e oito vértices.

No caso do modelo do gelo de seis vértices, construímos sua matriz de transferência de uma maneira bastante intuitiva e fizemos uma análise bastante completa de seus autovalores, em particular os autovalores maximais cuja importância nos é remetida pelo Teorema de Perron-Frobenius, concluindo que a transição de fase existe e ocorre em uma temperatura que depende das energias envolvidas no modelo. De fato, a $n$-ésima potência de sua matriz de transferência tem entradas nulas, para qualquer que seja o $n$, logo não há garantias de um único autovalor maximal por parte do Teorema de Perron-Frobenius.

No caso do modelo do gelo de oito vértices, construímos sua matriz de transferência e fizemos uma análise um pouco mais simplificada de seus autovalores. Em particular, a alternância da posição do autovalor maximal depende de um parâmetro $n$ associado a energia $\varepsilon_{4}$.

Os modelos do gelo, portanto, apresentam transição de fase unidimensional e tal transição está associada à degenerescência do seu autovalor máximo.

\section{Agradecimentos}

O autor agradece ao revisor anônimo e ao editor chefe pelas orientações e apontamentos, os quais qualificaram o trabalho.

\section{Material Suplementar}

O seguinte material suplementar está disponível online. Apêndice.

\section{Referências}

[1] R.A. Blythe e M.R. Evans, Brazilian Journal of Physics 33, 464 (2003).

[2] C.N. Yang e T.D. Lee, Physical Review 87, 404 (1952).

[3] C.N. Yang e T.D. Lee, Physical Review 87, 410 (1952).

[4] S. Friedli e Y. Velenik, Statistical Mechanics of Lattice Systems: A Concrete Mathematical Introduction (Cambridge University Press, Cambridge, 2017).

[5] S. Salinas, Introdução a Física Estatística (Edusp, São Paulo, 2018), $2^{\mathrm{a}}$ ed.

[6] L. Onsager, Physical Review 65, 117 (1944).

[7] L. Pauling, Journal of the American Chemical Society 57, 2680 (1935).

[8] J.C. Slater, Journal of the American Chemical Society 9, 16 (1941).

[9] R.J. Baxter, Physical Review Letters 26, 832 (1971).

[10] E.H. Lieb e F.Y. Wu, em: Two-dimensional ferroeletric models, editado por C. Domb e M.S. Green (Academic Press, London, 1972).

[11] J.A. Plascak e S.R. Salinas, Revista Brasileira de Física 10, 173 (1980).

[12] A. Ortiz-Ambriz, C. Nisoli, C. Reichhardt, C.J.O. Reichhardt e P. Tierno, Reviews of Modern Physics 9, 041003 (2019).

[13] M. Queffelec, Substitution Dynamical Systems - Spectral Analysis (Springer-Verlag, Berlin, 2010). 
[14] C.R. MacCluer, SIAM Review 42, 487 (2000).

[15] J.A. Cuesta e A. Sánchez, Journal of Statistical Physics 115, 869 (2004).

[16] T. Kato, Perturbation Theory for Linear Operators (Springer-Verlag, Berlim, 1980).

[17] E.H. Lieb, Physical Review Letters 18, 692 (1967).

[18] E.H. Lieb, Physical Review 162, 162 (1967).

[19] E.H. Lieb, Physical Review Letters 18, 1046 (1967).

[20] E.H. Lieb, Physical Review Letters 19, 108 (1967). 